\title{
EGitudios EGerenciales
}

Journal of Management and Economics for Iberoamerica

Articulo de investigación

\section{Caracterización de las pymes colombianas y de sus fundadores: un análisis desde dos regiones del país}

\author{
Mónica Franco-Ángel * \\ Directora Programa de Administración de Empresas, Facultad de Ciencias Administrativas y Económicas, Universidad Icesi, Cali, Colombia. \\ mfranco@icesi.edu.co
}

David Urbano

Profesor Asociado, Departamento de Empresa, Universidad Autónoma de Barcelona, Barcelona, España.

\section{Resumen}

Esta investigación tiene como objetivo realizar una caracterización de las pequeñas y medianas empresas (pymes) colombianas y de sus fundadores. Se realiza una investigación descriptiva con 384 empresas ubicadas en los departamentos de Cundinamarca y Valle del Cauca. Los resultados indican que en su mayoría las empresas superan los 48 meses de edad, cuentan en promedio con ventas anuales de hasta 5.000 millones de pesos colombianos, y que el tamaño de la planta de personal en la actualidad es hasta cinco veces el que tenían en el momento de su creación. En relación con los fundadores, estos superan los 36 años, presentan una sólida formación académica y se consideran buenos líderes.

Palabras clave: pymes, caracterización pymes, empresarios pymes.

\section{Characteristics of Colombian SMEs and their founders: an analysis in two regions of that country}

\section{Abstract}

This research aims to make a description of Colombian small and medium enterprises - SMEs- and their founders. We performed a descriptive research with 384 firms located in the regions of Cundinamarca and Valle del Cauca. The results indicate that most firms are over 48 months old, their average annual sales are up to 1,6 million dollars, and the size of their personnel is up to five times the original size. Their founders, in general, are older than 36 years old, have a solid academic background, and they consider themselves as good leaders.

Keywords: SMEs, characteristics of SMEs, entrepreneurs of SMEs.

\section{Caracterização das PMEs colombianas e seus fundadores: uma análise de duas regiões}

\section{Resumo}

O objetivo desta pesquisa é caracterizar as Pequenas e Medias Empresas (PMEs) da Colômbia e seus fundadores. Realiza-se uma investigação descritiva com 384 empresas localizadas nos departamentos de Cundinamarca e Valle del Cauca. Os resultados indicam que a maioria das empresas ultrapassa os 48 meses de idade, têm em média venda anuais de até 5.000 milhões de pesos colombianos, e que o tamanho da planta de pessoal é atualmente até cinco vezes o que tinham no momento de sus criação. Em relação aos fundadores, são maiores de 36 anos, possuem solida formação acadêmica e consideram-se bons líderes.

Palavras-chave: PMEs, caracterização PMEs, empreendedores de PMEs.

* Autor para dirigir correspondencia. Dirigir a: Universidad Icesi, Calle 18 No. 122-135, Cali-Colombia.

Clasificación JEL: M10, M21.

Cómo citar: Franco-Ángel, M. y Urbano, D. (2019). Caracterización de las pymes colombianas y de sus fundadores: un análisis desde dos regiones del país Estudios Gerenciales, 35(150), 81-91. https://doi.org/10.18046/j.estger.2019.150.2968

DOI: https://doi.org/10.18046/j.estger.2019.150.2968

Recibido: 29-may-2018

Aceptado: 19-mar-2019

Publicado: 29-mar-2019 


\section{Introducción}

Colombia es un país cuyo tejido empresarial está constituido, en su mayoría, por empresas de pequeña escala. Las pequeñas y medianas empresas (pymes)' ${ }^{1}$, definidas según las leyes colombianas como aquellas que poseen una planta de personal inferior a 200 empleados y activos totales de hasta 30.000 salarios mínimos mensuales legales vigentes $^{2}$, representan el 99,5\% del parque empresarial nacional (Murillo y Restrepo, 2016). Dado su tamaño y el papel clave que desempeñan, estas empresas son fundamentales para el desarrollo del país. En este sentido, las pymes son consideradas como la columna vertebral de las economías modernas, siendo vitales para sostener el crecimiento económico en el largo plazo (Ardic, Mylenko y Saltane, 2011), y con el potencial para estimular la expansión económica y actuar como estabilizadores en las recesiones (Varum y Rocha, 2013). Estudios previos demuestran la importancia de este tipo de empresas, en relación con la generación de empleo, el crecimiento del Producto Interno Bruto (PIB), la evolución de las reformas tecnológicas y la contribución de divisas para impulsar el crecimiento económico (Benzing, Chu y Orhan, 2009; Lussier y Halabi, 2010; Barbero, Casillas y Feldman, 2011).

Por otro lado, las pymes poseen una estructura más plana y menos burocrática, con menos procedimientos y sistemas formales, lo que facilita la rápida toma de decisiones y su capacidad de respuesta al mercado (Candiya, Mpeera, Munene y Akol, 2017). Cuentan con una organización interna más simple que les permite mayor adaptación al cambio (Lavia y Hiebl, 2015); relaciones activas con las partes interesadas, que se constituyen en sus fuentes de conocimiento más importante (Schweizer, 2013); y clima organizacional favorable dadas las relaciones cercanas, casi familiares, entre los empleados y los fundadores y directivos (Franco-Ángel y Urbano, 2010).

Sin embargo, estas empresas poseen debilidades intrínsecas a su dimensión, basadas en unos recursos propios restringidos, las cuales disminuyen su competitividad y limitan su crecimiento. Según varios estudios sobre el tema, estas debilidades se traducen en: poco acceso a fuentes de financiación (Karlan y Valdivia, 2011; Drexler, Fischer y Schoar, 2014); menor aprovechamiento de las economías de escala y escaso acceso a una amplia base de recursos (Burgstaller y Wagner, 2015; Lavia y Hiebl, 2015); carencia de habilidades de gestión en los empleados (Ates, Garango, Cocca y Bititchi, 2013; Yan, 2010) y poco acceso a personal con alto nivel educativo (Baporikar, Nambira y Gomxos, 2016); enfoque gerencial de corto plazo y poco desarrollo

En Colombia, la clasificación de las empresas como micro, pequeña, mediana y grande, está definida por la ley 590 de 2000, la cual fue modificada por las leyes 0905 de 2004 y 1450 de 2011 (artículo 43), la cual determina: empresa micro cuenta con una planta de personal no superior a 10 trabajadores y activos totales por valor inferior a 500 salarios mínimos mensuales legales vigentes. Empresa pequeña cuenta con una planta de personal entre 11 y 50 trabajadores y activos totales por valor entre 501 y 5.000 . Empresa mediana cuenta con una planta de personal entre 51 y 200 trabajadores y activos totales por valor entre 5.001 y 30.000 . Empresa grande cuenta con más de 200 empleados y activos mayores a 30.001 salarios mínimos mensuales legales vigentes (http://www. mipymes.gov.co/publicaciones/2761/definicion_tamano_empresarial_micro_pequena_ mediana_o_grande)

El salario mínimo en Colombia es de COP 828.116 equivalente a USD 265 al $16 / 03 / 2019$ de capacidades internas (Amer, Daim y Jetter, 2013); debilidad en el flujo de la información y en la efectividad de la comunicación (Temtime y Pansiri, 2005); dificultad para la consecución de clientes importantes y estrategia de mercadeo poco estructurada y reactiva para responder al mercado (Jones y Rowley, 2011; Franco, Santos, Ramalho y Nunes, 2014); escasa incorporación de la innovación en sus actividades (Madrid-Guijarro, Garcia-Perez-de-Lema y Van Auken, 2013), debido a escasas capacidades internas para detectar, asimilar e integrar el conocimiento externo (Spithoven, Vanhaverbeke y Roijakkers, 2013) y al poco acceso al conocimiento científico actualizado (Abouzeedan, Klofsten y Hedner, 2013); y carencia de sistemas de aseguramiento de la calidad por falta de comprensión de los principios de gestión de calidad y de tiempo de la alta dirección para impulsar los procesos (Mendes y Lourenço, 2014).

Las anteriores debilidades estructurales, sugeridas en la literatura, impiden que estas empresas enfrenten los retos que les presenta una nueva economía mundial más integrada e interdependiente. Para aumentar sus posibilidades de supervivencia y crecimiento, es evidente que estas empresas requieren de una estructura organizativa más sólida y un crecimiento sostenido (Segarra y Callejón, 2000).

Por todo lo anterior, es importante trabajar en la caracterización de estas empresas y de sus fundadores, ya que este conocimiento permitirá que se desarrollen políticas y programas diseñados según sus características y enfocados en el mejoramiento de su productividad y competitividad.

En este contexto, el propósito principal de la investigación es identificar las características más relevantes de las pymes colombianas y de sus fundadores, partiendo de estudios previos como los de Franco-Ángel y Urbano 12010 , 2014, 2016), Dini y Stumpo (2018) y Frohmann, Mulder y Olmos (2018).

Para alcanzar este objetivo, se realiza un estudio estadístico de tipo cuantitativo a partir de una muestra de 384 pymes ubicadas en los departamentos de Cundinamarca y del Valle del Cauca, las cuales constituyen dos de las regiones más representativas a nivel empresarial ya que concentran el $42 \%$ de las empresas del país y agrupan un gran número de las empresas formales (Murillo y Restrepo, 2016). La información se recolecta mediante una encuesta estructurada que se envía a los gerentes/fundadores de las empresas por correo postal y electrónico, obteniendo un nivel de respuesta final, por parte de los empresarios, del $20 \%$.

Con este trabajo se espera realizar una contribución, tanto desde la perspectiva académica, porque permite un mayor conocimiento de las características de las pymes colombianas y de sus fundadores, como desde el plano gubernamental, en relación con el diseño de políticas de apoyo a las empresas.

El presente artículo consta de cinco apartados. Después de la introducción, se presenta el estado de la cuestión sobre la caracterización de las pymes en Colombia, luego se aborda la metodología utilizada y el diseño de la investigación, y más adelante se presentan los resultados obteni- 
dos. Al final, se encuentran las conclusiones generales que apuntan a lo que se considera más relevante del estudio, y sus implicaciones.

\section{Marco teórico}

Aunque en América Latina las pymes representan el $99,5 \%$ del total de las empresas y son responsables del $61 \%$ del empleo formal (Correa, Leiva y Stumpo, 2018), solo contribuyen con el 30\% del PIB de la región (Casaburi, Suaznábar y Llisterri, 2016); las más pequeñas de estas empresas corresponden al $89 \%$ y contribuyen con solo el $29 \%$ del empleo formal (Correa et al., 2018), lo que muestra la gran brecha de productividad que existe entre estas y las grandes empresas, las cuales constituyen el $0,5 \%$ de las empresas del país y en contraste aportan el $70 \%$ del PIB de la región.

En la región, las pymes presentan pocos avances en procesos de innovación y un atraso tecnológico considerable, lo que reduce su potencial competitivo en los mercados. Frohmann et al. (2018) aducen que en América Latina el esfuerzo de las pymes por innovar es bajo, y que su vulnerabilidad no les permite incorporar la innovación como una de sus estrategias. Por otra parte, Crespi, Tacsir y Vargas (2016) evidencian que la innovación está relacionada con el tamaño, las capacidades y las características de las empresas, encontrando en su estudio mayor innovación en las empresas medianas que en las pequeñas.

Otra de las vulnerabilidades de las pymes en Latinoamérica es el poco acceso al crédito, especialmente para las empresas más pequeñas, lo cual, según Llisterri y GarcíaAlba (2008) sigue siendo uno de los principales obstáculos que enfrenta el desarrollo de estas empresas. Por su parte, Presbitero y Rabellotti (2016) reportan que menos del 15\% de los préstamos en la región va a empresas pequeñas.

Por otro lado, aunque en los diferentes países que conforman la región se ha realizado un esfuerzo por diseñar medidas de apoyo para las pymes, el fomento gubernamental para que estas empresas mejoren su productividad y competitividad sigue siendo muy débil, debido a la atomización de las actividades y a su enfoque de corto plazo. Correa, Dini y Stumpo (2018) confirman que en la región faltan entidades públicas especializadas con estrategias de largo plazo, y la participación de los empresarios en la creación de las políticas de apoyo.

Las pymes en Colombia, país que pertenece a la región, presentan una gran heterogeneidad no solo en su productividad sino también en las características de sus fundadores. Se encuentran desde empresas pequeñas centradas en actividades de baja productividad, que dependen de la demanda interna, orientadas por el pensamiento estratégico de su fundador, sin procesos de innovación y con poca o nula visión internacional; hasta empresas medianas de rápido crecimiento, dinámicas, innovadoras y exportadoras.

En este sentido, el Ministerio de Comercio, Industria y Turismo (2018) reporta que la producción de una empresa grande promedio en Colombia es siete veces mayor a la producción de una pyme promedio. Según el documento
CONPES 3866 de 2016 del Departamento Nacional de Planeación - DNP- (2016), una de las razones de la baja productividad en Colombia es la disminución en el número de actividades económicas y productos en los que el país es competitivo, especialmente en aquellos de mayor complejidad que son producidos y exportados.

En lo que respecta a innovación y tecnología, en comparación con Latinoamérica, Colombia se ubica por debajo del promedio de la región, y solo por encima del promedio en productos primarios (Blyde, 2014), siendo muy baja o inexistente la inversión en procesos de innovación y tecnología. De acuerdo con la Encuesta de Desarrollo e Innovación Tecnológica- EDIT- (2015-2016), solo el 21,5\% de las empresas encuestadas son innovadoras en sentido amplio y el $75 \%$ de estas no innovan. Estas empresas aducen que las principales razones para no innovar son la escasez de recursos propios y las dificultades para acceder a financiamiento externo a la empresa. Del capital invertido por estas empresas en actividades científicas, tecnológicas y de innovación en el año 2016 , solo el $20 \%$ corresponde a pymes. Esta misma encuesta muestra que únicamente el $6,5 \%$ de las empresas tuvieron registros de propiedad intelectual durante 2015-2016, entre los que predominaron los signos distintivos y marcas $(92 \%)$, los derechos de autor (3\%), los registros de diseños industriales $(2 \%)$ y las patentes de invención $(1,5 \%)$. Dichos resultados son confirmados por Escandón-Barbosa, Arias, Medina, Gómez, Varela, Martínez y Parra (2016), quienes aducen que el $70 \%$ de las empresas colombianas utiliza tecnologías de más de 5 años y el $82 \%$ de las nuevas empresas no usa tecnología de punta en sus procesos productivos.

Las empresas con mayor innovación en Colombia han sido objeto de estudio y reconocidas por sus avances en esta materia. Es así, como Tarapuez, Guzmán y Parra (2016), en su investigación sobre las empresas ganadoras del premio Innova del Ministerio de Comercio, Industria y Turismo de Colombia, durante el periodo 2010-2013, encuentran que las empresas que se relacionan con el entorno cuando abordan los procesos de investigación y desarrollo, y que contemplan en su plan estratégico el tema de la innovación, tienen mejores resultados en licenciamiento de patentes y en sus utilidades.

Por otra parte, las empresas colombianas presentan bajos niveles de aseguramiento de la calidad, tal como se confirma con la EDIT (2015-2016), en la que únicamente el $11 \%$ de las empresas manifestaron tener certificaciones de calidad en proceso y el $5 \%$ certificaciones de calidad de producto. De estas empresas el $24 \%$ y el $10 \%$, respectivamente, son pymes. Sin embargo, Calderón, Álvarez y Naranjo (2009) en un estudio en empresas industriales colombianas encuentran que al momento de establecer su estrategia, para las empresas son muy importantes la calidad del producto y el establecimiento de procedimientos de calidad del mismo.

Finalmente, la información reportada por el Banco Mundial (2018) evidencia que las pymes en Colombia son empresas jóvenes con un promedio de antigüedad de 17 años, siendo la mayoría de ellas dirigidas por hombres, con una 
participación de las mujeres en la gerencia del 23,2\% para las pequeñas y del $12,7 \%$ para las medianas; la experiencia del gerente en la industria es de 23 años; y la proporción de trabajadores no calificados (de todos los trabajadores de la producción) es de $25,6 \%$ para las pequeñas y de $38,7 \%$ para las medianas; y en cuanto al nivel educativo, Gómez y Borda (2018) concluyen que el $55 \%$ de los empleados de las pymes cuenta con educación secundaria y solo el $11 \%$ con título universitario.

\section{Metodología y diseño de la investigación}

En la presente investigación se realiza un análisis estadístico cuantitativo a partir de una muestra de empresas colombianas ubicadas en las ciudades de Bogotá, Soacha, Chía y Girardot (departamento de Cundinamarca), y en Cali, Tuluá, Palmira, Buga y Buenaventura (departamento del Valle del Cauca). Las empresas son seleccionadas de la base de datos NIT (Número de Identificación Tributario), elaborada por la empresa Dirnafax ubicada en la ciudad de Bogotá, dado que contiene información actualizada generada por las diferentes Cámaras de Comercio que operan en los departamentos mencionados, los cuales, según Murillo y Restrepo (2016), concentran el 33,1\% y el 8,9\%, del total de las empresas colombianas, respectivamente, es decir, el $42 \%$ del total de la población empresarial colombiana; además, solamente entre Bogotá y Cali se generó en 2015, el 40\% de la producción bruta del país (Departamento Administrativo Nacional de Estadística -DANE-, 2016).

En este orden de ideas, según las estadísticas del Censo del año 2005 y proyecciones al 2017 del DANE (2017), el número total de pymes que se encuentran en las ciudades seleccionadas, al 2015, asciende a 22.493. Por otra parte, el número de empresas registradas en las diferentes Cámaras de Comercio colombianas, en dichas ciudades, en el mismo año, es de 19.452. Por lo tanto, el tamaño de la población se toma de este último dato, ya que constituye el número de empresas registradas formalmente en el país. Por otra parte, para la selección de la muestra se utiliza un muestreo aleatorio estratificado con asignación proporcional, y se define un margen de error deseado del $5 \%$, y un nivel de confianza del 95\%. Dado lo anterior, y de acuerdo a los cálculos, el tamaño de la muestra corresponde a 384 empresas.

Los resultados presentados en este artículo hacen parte de una investigación previa de los autores sobre el dinamismo de las pymes en Colombia, en la cual se define como empresa dinámica aquella que haya tenido un crecimiento anual promedio en ventas del $20 \%$ en los últimos 5 años de existencia; en dicha investigación, se definen las variables de estudio en función de los recursos internos y externos que impactan el desempeño de las empresas. Entre estas variables se encuentran las utilizadas en este artículo, las cuales permiten la caracterización tanto de las empresas como de los empresarios.

Antes de la aplicación del cuestionario se realizó un pretest a 20 empresas, lo que permitió hacer ajustes al instrumento. Estas encuestas no hacen parte de la muestra final del estudio. Una vez ajustada, la encuesta se envía por correo postal y electrónico a los gerentes/fundadores de las empresas seleccionadas. Las variables son dicotómicas y ordinales. Estas últimas medidas a través de una escala de Likert de 5 puntos, donde 1 es el valor más bajo otorgado al factor y 5 el más alto. Dado el bajo nivel de respuesta, se enviaron 1.921 encuestas por correo físico (cinco veces el número requerido), para obtener después de varios intentos, 384 respuestas con la información completa, es decir, se obtuvo una tasa de respuesta final del $20 \%$.

\section{Resultados y discusión}

El tejido empresarial colombiano está compuesto en su mayoría por empresas de tamaño micro. Según Murillo y Restrepo (2016), del total de las empresas colombianas el $92,3 \%$ son microempresas y el $7,2 \%$ son pymes, y se concentran en los sectores comercio $(42 \%)$ y servicios $(40 \%)$ (tabla 1).

Las empresas de esta investigación son en su mayoría establecidas (91\%), las cuales según el estudio Global Entrepreneurship Monitor son aquellas que tienen 42 meses o más de creadas; en su mayoría son pequeñas según su tamaño (81\%), predominando las empresas pertenecientes al sector industrial (59\%). Adicionalmente, el 78,8\% de las empresas presentan un nivel de ventas de hasta 5.000 millones de pesos anuales (figura 1). Por otra parte, el estudio muestra que el $13 \%$ de las empresas ha disminuido sus ventas durante los últimos 5 años, y que la mayoría las ha incrementado entre $1 \%$ y $19 \%$, presentando aumentos superiores al $20 \%$ solo el $21 \%$ de las empresas.

En lo que se refiere al crecimiento de la planta de personal en las empresas, se observa que más de la mitad de estas presentan incrementos de por lo menos 3 veces su tamaño inicial (figura 2), lo que confirma su importancia en cuanto a la generación de empleo, tal como lo han evidenciado el Banco Mundial (2015a) y Correa et al. (2018), quienes confirman que las pymes formales contribuyen con el $60 \%$ del empleo total.

Frente a la formación académica de su personal se encuentra que solo el $17 \%$ tiene estudios universitarios y el $2 \%$ de postgrado (figura 2), lo que refleja un bajo nivel de profesionalización en las empresas, esto coincide con el estudio de Gómez y Borda (2018). Por su parte, Baporikar et al. (2016) reconocen como una debilidad de las pymes su poco acceso a personal con altos niveles de educación, dado que sus recursos restringidos no les permite el pago de salarios competitivos en el mercado.

En cuanto a los aspectos relacionados con el sector económico, los empresarios consideran como muy importantes: la especialización (30\%), seguido de la competencia $(18 \%)$ y la tecnología (17\%) (figura 3). Estos hallazgos se confirman con las investigaciones realizadas por Kunc y Morecroft (2010) y Middleton y Chambers (2010), en las cuales se reconoce el impacto del sector económico en el desempeño de las empresas.

Referente a los factores externos que impactan el dinamismo de la empresa, el apoyo y la aceptación por parte 
Tabla 1. Clasificación de las empresas según tamaño y sector económico en 2015.

\begin{tabular}{lllllll}
\hline $\begin{array}{l}\text { Tamaño de la } \\
\text { empresa }\end{array}$ & $\begin{array}{l}\text { No. de } \\
\text { Empresas }\end{array}$ & $\begin{array}{l}\text { Participación del total de } \\
\text { empresas }\end{array}$ & $\begin{array}{l}\text { Sector } \\
\text { comercio }\end{array}$ & $\begin{array}{l}\text { Sector } \\
\text { servicios }\end{array}$ & $\begin{array}{l}\text { Sector Industria } \\
\text { Cector } \\
\text { Construcción }\end{array}$ \\
\hline Grande & 6.361 & $0,5 \%$ & $0,1 \%$ & $0,2 \%$ & $0,1 \%$ & $0,1 \%$ \\
Mediana & 19.980 & $1,4 \%$ & $0,3 \%$ & $0,7 \%$ & $0,2 \%$ & $0,2 \%$ \\
Pequeña & 79.926 & $5,8 \%$ & $1,6 \%$ & $2,7 \%$ & $0,9 \%$ & $0,8 \%$ \\
Microempresa & 1.273 .017 & $92,3 \%$ & $39,9 \%$ & $36,4 \%$ & $10,9 \%$ & $12 \%$ \\
Total & 1.379 .284 & $100 \%$ & $42 \%$ & $40 \%$ & $5,0 \%$ & $6 \%$ \\
\hline
\end{tabular}

Fuente: elaboración propia a partir de Murillo y Restrepo (2016).

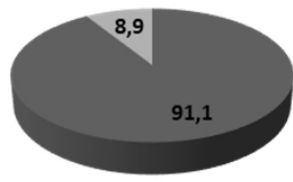

a)

c)
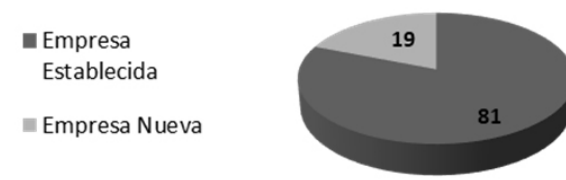

b)
- Pequeñas

m Mediana

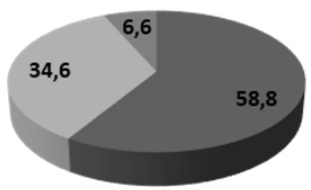

\section{- Industria \\ Comercio}

- Servicios

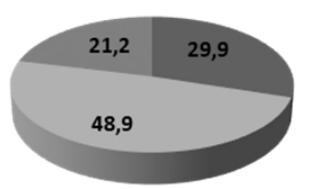

- 0-1.000

$=1.000-5.000$

$=5.000$

d)

Figura 1. Porcentaje de las empresas según edad (a), tamaño (b), sector económico (c) y nivel de ventas en millones de pesos colombianos (d), en 2015.

Fuente: elaboración propia.

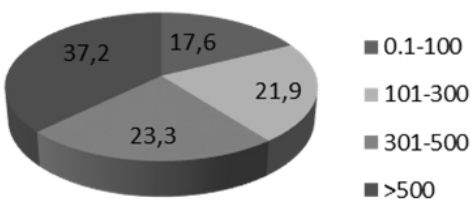

a)

Figura 2. Situación de la planta de personal de las empresas en cuanto a crecimiento lal y nivel educativo (b), en 2015 (datos en porcentajes).

Fuente: elaboración propia. de la comunidad es el factor que los empresarios consideran como el más importante (74\%), seguido en su orden por el apoyo de la familia (65\%) y la situación del sector económico (63\%) (figura 4). Lo anterior coincide con lo que evidencia la literatura, en cuanto al impacto que los factores externos o del entorno presentan en el desempeño de las empresas. Por su parte, autores como Álvarez y Urbano (2011); Álvarez, Urbano, Coduras y Ruiz-Navarro (2011); y Welter y Smallbone (2011), confirman la relevancia de los factores del entorno como condicionantes de la creación de las empresas.

En cuanto a los factores que dificultan el dinamismo de las empresas, los empresarios entrevistados mencionan como los más relevantes la falta de preparación empresarial $(80 \%)$, la competencia $(79 \%)$, la falta de capital inicial
(79\%) y la inexperiencia del empresario (76\%) (figura 5).

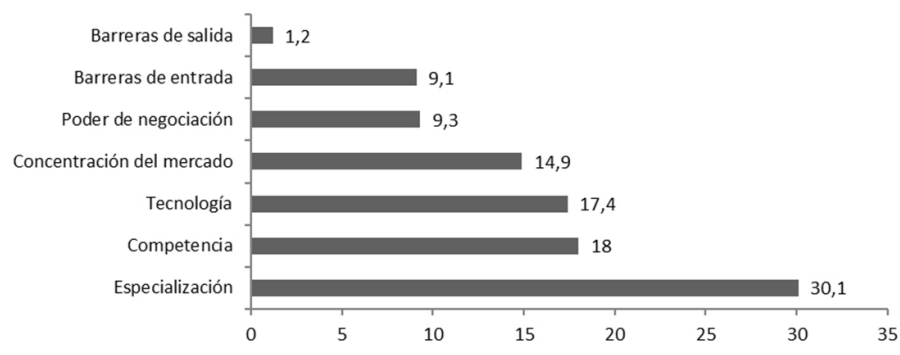

Figura 3. Importancia de los aspectos del sector económico al que pertenecen las empresas, en 2015 (datos en porcentajes).

Fuente: elaboración propia. 
Por otra parte, las entidades a las que con mayor frecuencia recurren los empresarios para buscar apoyo son: los bancos (88\%) y las Cámaras de Comercio (81\%) (figura 6). Además, los empresarios consideran que los bancos son las entidades que más han contribuido al desarrollo de sus empresas. En línea con estos resultados, Bouri, Breij, Diop, Kempner, Klinger y Stevenson (2011) aclaran que el acceso al sector financiero es necesario para crear un entorno económico que permita a las empresas crecer y prosperar. Así mismo, un estudio del Banco Mundial (2015b) indica que el acceso a la financiación mejora el rendimiento y el crecimiento de las empresas facilitando la entrada al mercado, la reducción del riesgo, la promoción de la innovación y la actividad empresarial en las economías en desarrollo.

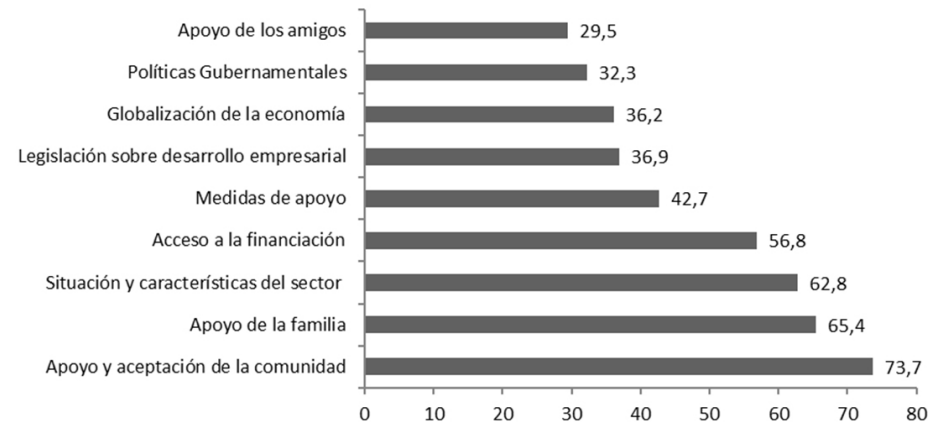

Figura 4. Importancia de los factores externos en el dinamismo de las empresas, en 2015 (datos en porcentajes).

Fuente: elaboración propia.

En relación con las medidas de apoyo, el crédito bancario es al que más recurren los empresarios ( $86 \%$ ), y las subvenciones ( $1 \%)$, las incubadoras $(3 \%)$ y el capital de riesgo $(4 \%)$, las menos tenidas en cuenta (figura 7). Así mismo, los empresarios consideran que los créditos bancarios son las medidas que más han contribuido al dinamismo de sus empresas, a pesar de que estudios previos evidencian el poco acceso de las pymes al financiamiento (Presbitero y Rabellotti, 2016). Por esta razón, algunas pymes satisfacen sus necesidades financieras recaudando capital de sus familias, amigos o conocidos (Hussain y Matlay, 2007), mientras que otras se financian con bancos comerciales u otras instituciones financieras (Song y Wang, 2013).

Adicionalmente, a través del presente estudio se comprueba que los fundadores conceden gran valor a su profesión de empresario (76\%) y están seguros del impacto positivo que sus empresas generan en la comunidad (86\%). También, se evidencia que la mayoría de los empresarios considera que la comunidad ve su profesión como signo de prestigio (88\%), que un buen número de ellos es consciente que la comunidad reconoce la importancia de las pymes para el desarrollo económico de la región y del país (89\%), y que la imagen de sus empresas frente a la comunidad es positiva (figura 8); esto es coherente con lo que se indica en un estudio previo de Franco-Ángel y Urbano (2010).

La mayoría de los empresarios considera que la cultura organizativa impacta sus empresas positivamente (76\%), y dos terceras partes piensan lo mismo respecto a la cultura regional (61\%) (figura 9), tal como lo han confirmado los

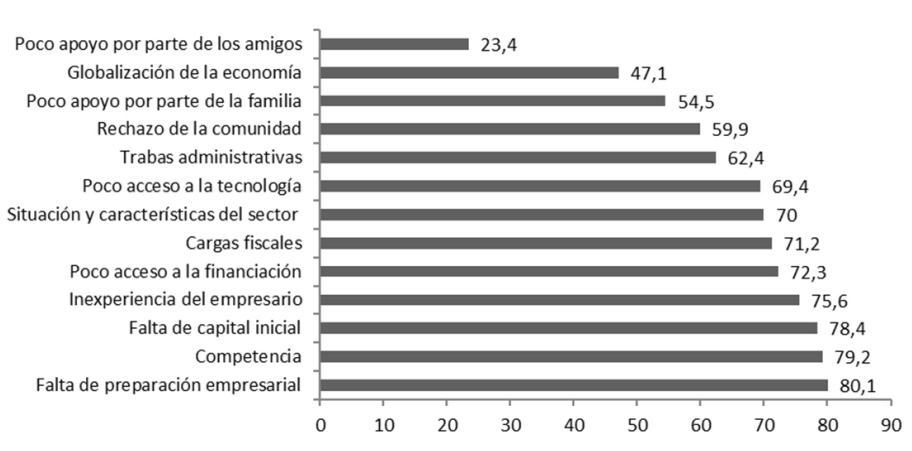

Figura 5. Factores que dificultan el dinamismo de las empresas, en 2015 (datos en porcentajes).

Fuente: elaboración propia.

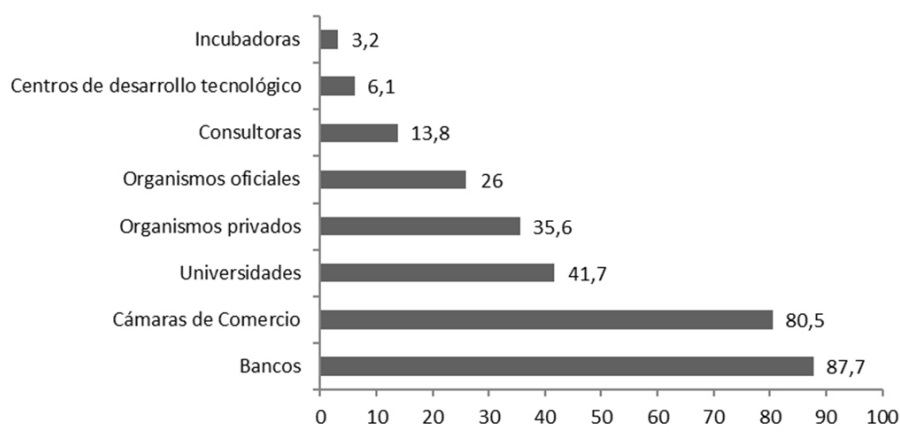

Figura 6. Entidades de apoyo utilizadas por los empresarios, en 2015 (datos en porcentajes).

Fuente: elaboración propia.

estudios de Swoboda, Meierer, Foscht y Morschett, (2011) y Tey e Idris (2012).

Estudios empíricos llevados a cabo por Chatzoglou, Chatzoudes, Sarigiannidis y Theriou (2018), Kamasak (2017) y Haji y Ghazali (2018) permiten confirmar la importancia de los factores internos (propios de la empresa) en el desempeño de las empresas. En esta investigación, la reputación y el prestigio de la empresa (91\%), seguido por el conocimiento, habilidades, destrezas $(90 \%)$ y características personales del equipo fundador (89\%) (figura 10), son los factores internos que los empresarios consideran como los de mayor relevancia. Según Soini y Veseli (2011), el conocimiento del negocio por parte del fundador tiene un impacto en su crecimiento, y las habilidades de gestión, el conocimiento y la experiencia previa también contribuyen

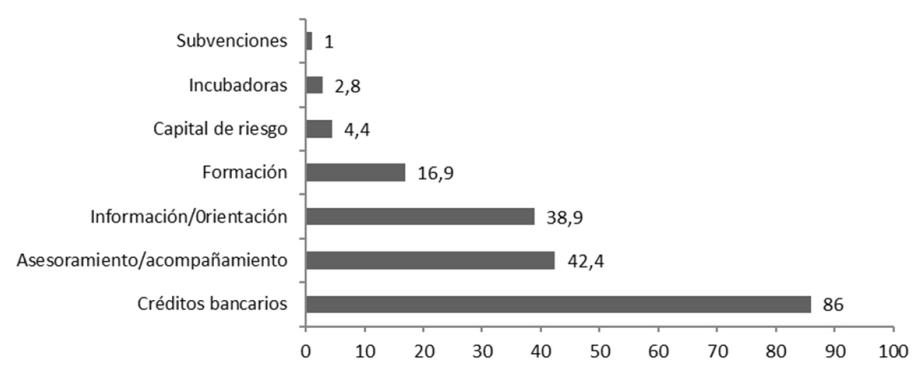

Figura 7. Medidas de apoyo utilizadas por los empresarios, en 2015 (datos en porcentajes).

Fuente: elaboración propia. 


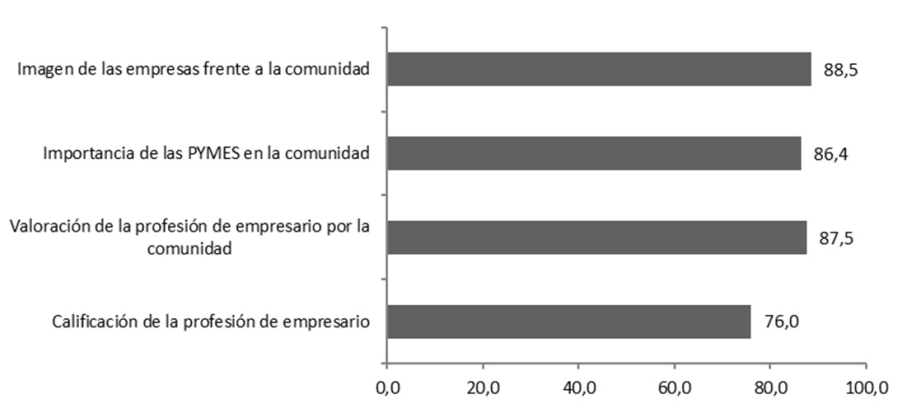

Figura 8. Importancia de las pymes y de los empresarios, en 2015 (datos en porcentajes).

Fuente: elaboración propia.

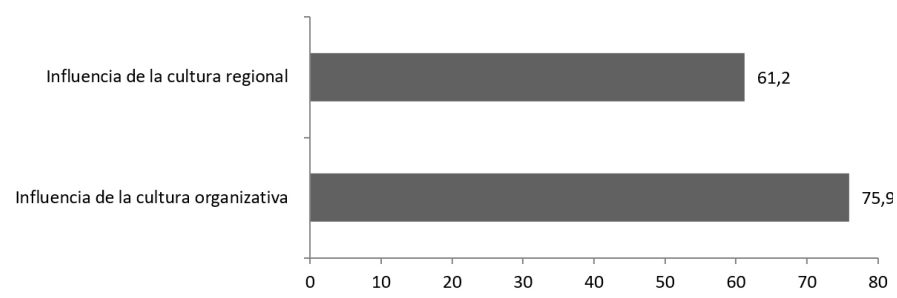

Figura 9. Influencia positiva de la cultura regional y organizacional, en 2015 (datos en porcentajes).

Fuente: elaboración propia.

al rendimiento y crecimiento de la empresa. Adicionalmente, Rasmussen y Nielsen (2011) y Sidik (2012) evidencian que un nivel más alto de formación empresarial tiene un efecto positivo en la productividad, lo que posteriormente conduce a una mayor rentabilidad.

Algunos autores reconocen la importancia del desarrollo de capacidades organizativas en el interior de las empresas, y las definen como la habilidad para utilizar recursos a través de los procesos con el objetivo de obtener un fin determinado (Amit y Schoemaker, 1993), y como una rutina o un número de rutinas que interactúan juntas (Grant, 1991), y las relacionan con las habilidades específicas de la empresa, el conocimiento y los valores que son acumulados a través del aprendizaje y del entrenamiento en el trabajo (Dierickx y Cool, 1989). En este orden de ideas, en las empresas estudiadas, las capacidades organizativas calificadas como las más importantes son las desarrolladas en el área de producción (90\%), seguidas de las desarrolladas en el área administrativa (85\%) y en el área de mercadeo (85\%) (figura 11).

El perfil del empresario se define a partir de variables como la edad, el nivel educativo, la formación empresarial antes de crear la empresa actual, la experiencia previa en creación de otras empresas y en el sector económico al que pertenece la empresa actual. Cuentan de igual forma, las características y habilidades, así como la presencia de padres y parientes empresarios. Es este estudio, los empresarios, en general, son mayores de 36 años (82\%) y tienen un nivel de educación alto $(71 \%)$; más de la mitad recibió formación empresarial antes de crear la empresa actual $(57 \%)$, y más de una tercera parte de ellos posee experiencia previa en la industria a la que pertenece su empresa (46\%) (figura 12).

En lo que se refiere a las características personales, los empresarios consideran como las más relevantes para el éxito de su empresa, la confianza en sí mismos (15\%), seguida de la visión de futuro (13\%) (figura 13). Complementando lo anterior, investigadores como Adomako, Danso, Uddin y Damoah (2016) encuentran en su estudio que las diferencias en el desempeño de las pymes están relacionadas con las características de la personalidad de los empresarios.

Casi la mitad de los empresarios afirma que sus padres son o fueron empresarios (48\%), y la mitad de estos que tiene parientes empresarios diferentes a sus padres (50\%). De la misma manera, la mayoría aduce haber recibido el apoyo de sus familias en el proceso empresarial (85\%) y más de la mitad afirma haber recibido el apoyo de sus amigos (57\%) (figura 14).

De otra parte, los resultados de la presente investigación muestran que la participación en redes, tanto de las empresas como de los empresarios, es poca. Menos de la mitad de las empresas $(40 \%)$ y un poco más de la tercera parte de los empresarios (39\%) ha participado en las mismas (figura 15). A pesar de que muchos estudios confirman la importancia de la asociatividad para las pymes que desean ingresar exitosamente a mercados más amplios y adquirir recursos complementarios (Lindstrand, Melen y Nordman, 2011; Kontinen y Ojala, 2011), la disposición de los empresarios colombianos a la misma es baja, dado el temor que existe al oportunismo que pueda presentarse (Franco-Ángel y Urbano, 2014).

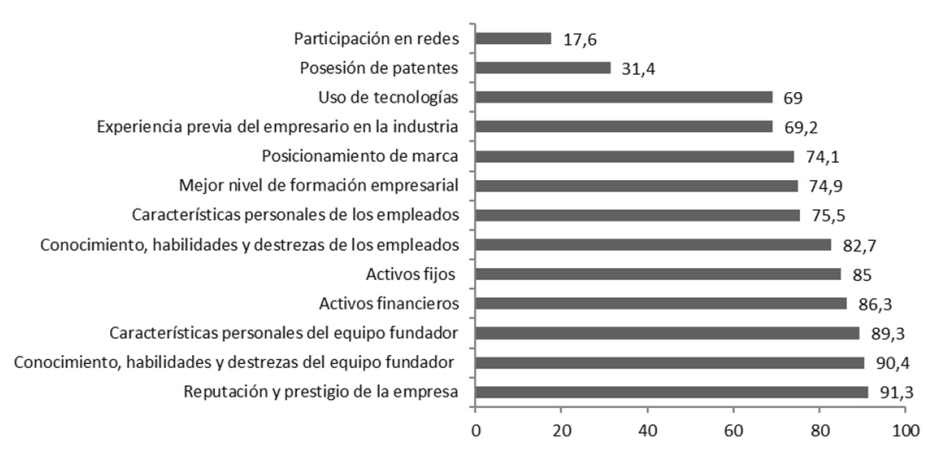

Figura 10. Impacto de factores internos en el dinamismo de las empresas, en 2015 (datos en porcentajes).

Fuente: elaboración propia.

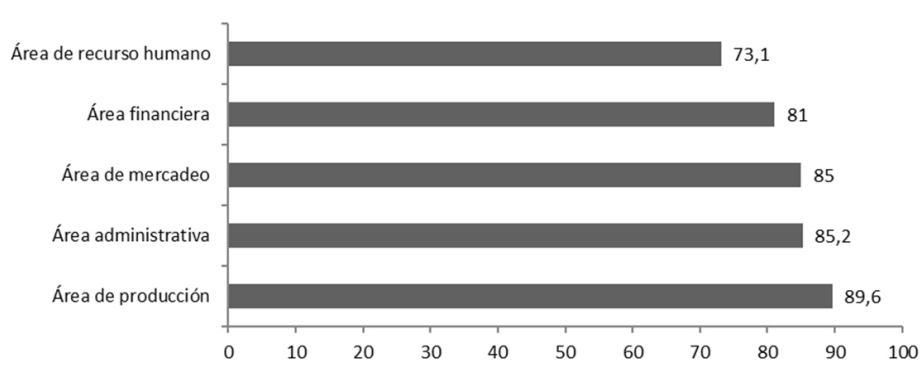

Figura 11. Impacto de capacidades organizativas en el dinamismo de las empresas, en 2015 (datos en porcentajes).

Fuente: elaboración propia. 


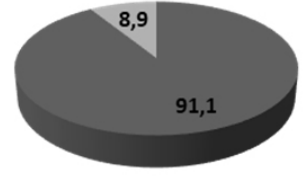

a)

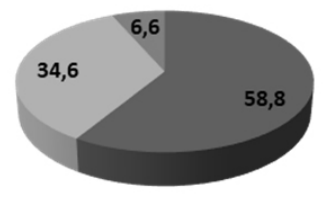

c)

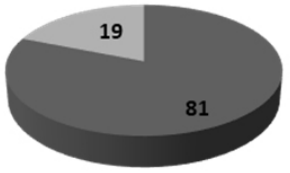

- Pequeñas

Mediana

b)

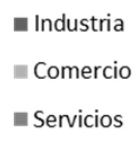

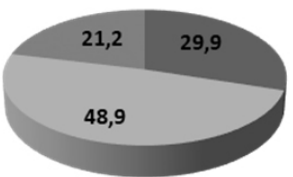

- 0-1.000

$=1.000-5.000$

$=5.000$

Figura 12. Perfil de los empresarios en cuando a edad (a), nivel educativo (b), formación empresarial (c) y experiencia previa en creación de empresas y en el sector económico (d), en 2015 (datos en porcentajes).

Fuente: elaboración propia.

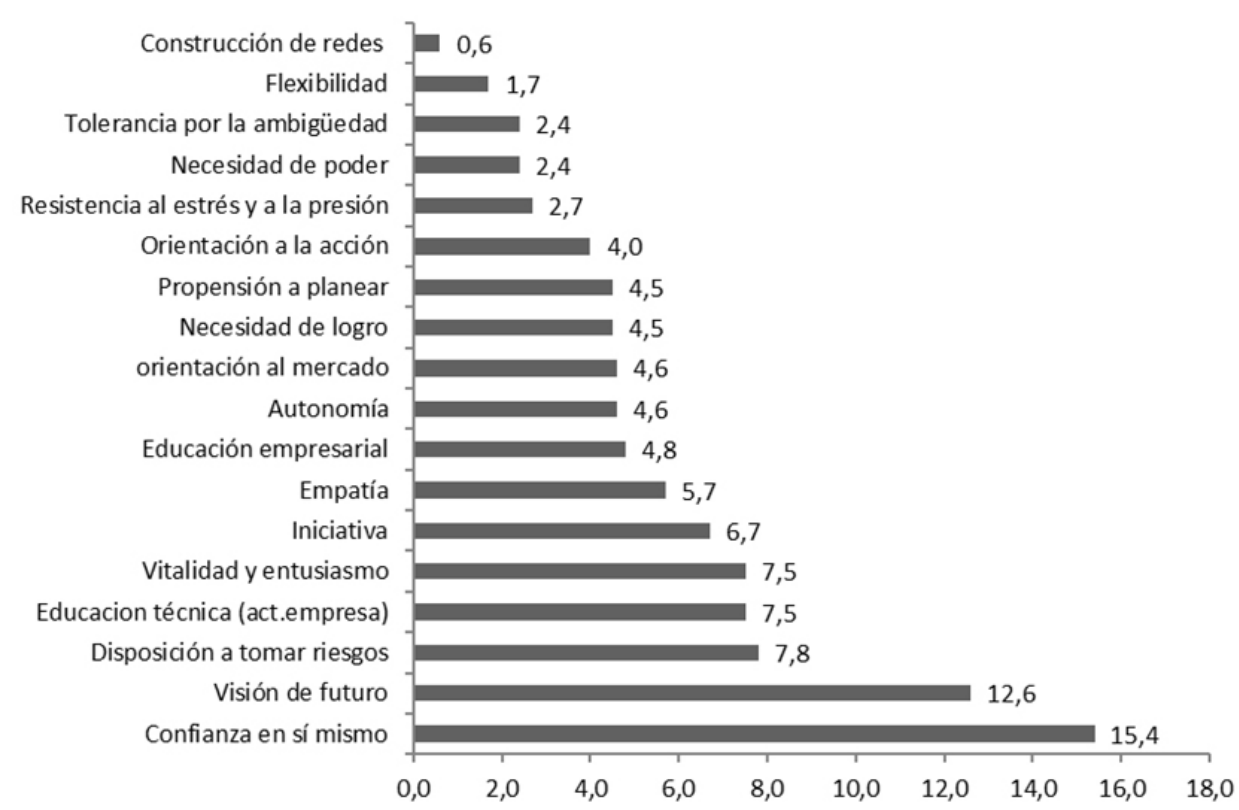

Figura 13. Características de los empresarios, en 2015 (datos en porcentajes).

Fuente: elaboración propia.

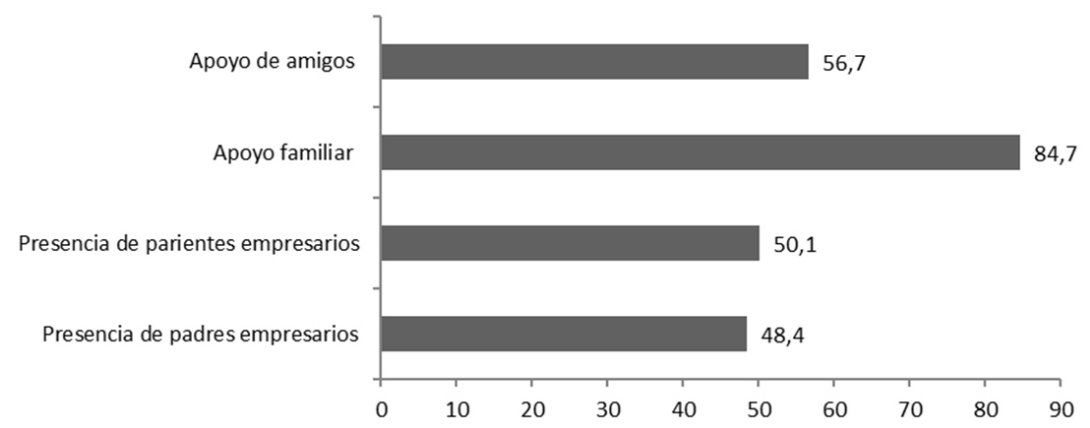

Figura 14. Presencia de padres y parientes empresarios y apoyo de la familia y de los amigos, en 2015 (datos en porcentajes).

Fuente: elaboración propia. 


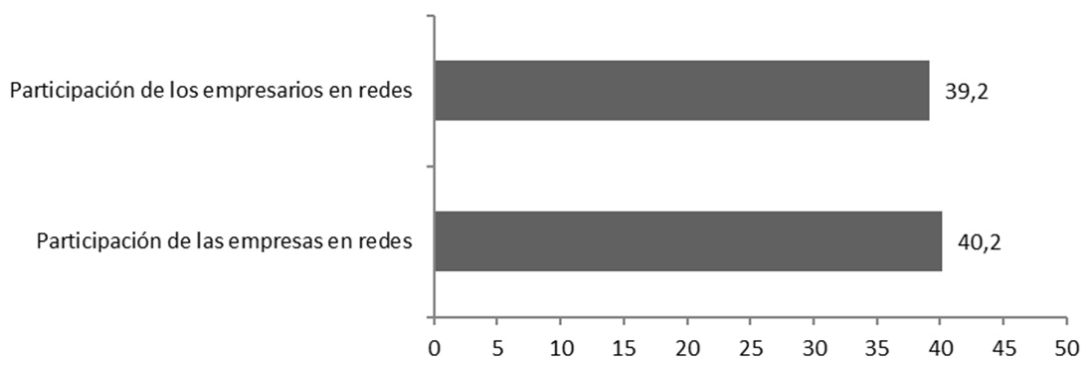

Figura 15. Participación de la empresa y de los empresarios en redes, en 2015 (datos en porcentajes). Fuente: elaboración propia.

\section{Conclusiones}

El estudio permite identificar las características más relevantes de las pymes estudiadas y de sus fundadores, algunas de las cuales coinciden con estudios previos sobre la pyme en América Latina y particularmente en Colombia.

Los resultados obtenidos sugieren que los empresarios de las pymes deben conceder mayor importancia a la construcción de la reputación y del prestigio de su empresa en el mercado, y a lograr el apoyo y la aceptación por parte de la comunidad, en aras de lograr un mayor dinamismo. Así mismo, y ante la importancia que revisten el conocimiento, las habilidades y las destrezas de los empleados, estas empresas deben trabajar por mejorar el bajo nivel educativo de sus empleados e incrementar su profesionalización.

Por su parte, las instituciones gubernamentales que apoyan a las pymes, al igual que las instituciones de desarrollo tecnológico, instituciones financieras, Cámaras de Comercio, gremios y universidades, entre otros, pueden diseñar programas que propendan por mejorar la preparación empresarial de los empresarios; incrementar su acceso a las fuentes de financiación y a alternativas diferentes al crédito bancario; disminuir las trabas administrativas para la creación de las empresas; diseñar esquemas especiales de contribución tributaria; y fomentar la transferencia, el uso de tecnología y la especialización en los sectores económicos.

Así, partiendo de las características propias de las pymes colombianas, dichas instituciones y entidades podrán soportar el desarrollo de estas empresas y las empoderarán para responder mejor a su entorno.

Considerando que este estudio se constituye en una aproximación a la definición del perfil de las empresas pymes en Colombia y de sus fundadores, y a la identificación de factores internos y externos que impactan su dinamismo, quedan muchas preguntas sin responder, lo que sugiere la realización de investigaciones que permitan un mayor acercamiento a la problemática de esta tipología de empresas, y que generen mayor conocimiento sobre las mismas. De igual manera, se reconoce que este estudio tiene un gran componente subjetivo pues está basado en las percepciones de los fundadores de las empresas investigadas.

En cuanto a futuras líneas de investigación, se considera importante realizar estudios de tipo longitudinal, con el fin de conocer los cambios que se presentan en el perfil de estas empresas y de sus fundadores, y se sugiere la realización de un mayor número de investigaciones de tipo cualitativo, con el fin de profundizar en el impacto que dicho perfil tiene en el desempeño de las mismas.

\section{Financiación}

En la elaboración de la investigación y del presente artículo se recibió el apoyo financiero de la Universidad Icesi (Colombia).

\section{Nota}

Artículo derivado de la tesis doctoral "Factores determinantes del dinamismo de las pymes en Colombia". Doctorado Internacional en Creación y Gestión de Empresas. Universidad Autónoma de Barcelona, Barcelona, España. Los datos usados en este artículo han sido actualizados al año 2015.

\section{Conflicto de intereses}

Los autores declaran no tener ningún conflicto de intereses.

\section{Bibliografía}

Abouzeedan, A., Klofsten, M. y Hedner, T. (2013). Internetization management as a facilitator for managing innovation in High-Technology smaller firms. Global Business Review, 14(1), 121-136. https://doi.org/10.1177/0972150912466462

Adomako, S., Danso, A., Uddin, M. y Damoah, J. (2016). Entrepreneurs' optimism, cognitive style and persistence. International Journal of Entrepreneurial Behavior \& Research, 22(1), 84-108. https://doi.org/10.1108/IJEBR-07-2015-0158

Álvarez, C. y Urbano, D. (2011). Environmental factors and entrepreneurial activity in Latin America. Academia Revista Latinoamericana de Administración, 48(1), 126-139.

Álvarez, C., Urbano, D., Coduras, A. y Ruiz-Navarro, J. (2011). Environmental conditions and entrepreneurial activity: a regional comparison in Spain. Journal of Small Business and Enterprise Development, 18(1), 120-140. https://doi.org/10.1108/14626001111106460

Amer, M., Daim, T.U. y Jetter, A. (2013). A review of scenario planning. Futures, 46, 23-40. http://dx.doi.org/10.1016/j.futures.2012.10.003

Amit, R. y Schoemaker, P.JH. (1993). Strategic assets and organizational rents. Strategic Management Journal, 14(1), 33-46.

Ardic, O.P., Mylenko, N. y Saltane, V. (2011). Small and medium enterprises: a cross-country analysis with a new data set. The World Bank Policy Research, Working Paper 5538, 1(1), 1-30. https://doi. org/10.1111/j.1468-0106.2012.00596.x

Ates, A., Garango, P., Cocca, P. y Bititci, U. (2013). The development of 
SME managerial practice for effective performance management. Journal of Small Business and Enterprise Development, 20(1), 28-54. https://doi.org/10.1108/14626001311298402

Banco Mundial. (2015a). Doing Business 2014: Understanding Regulations for Small and Medium-Size Enterprises. Recuperado el 20 de mayo de 2018 de: http://www.doingbusiness.org/ /media/WBG/DoingBusiness/Documents/Annual-Reports/English/DB14-Full-Report.pdf

Banco Mundial. (2015b). Small and Medium Enterprises (SMEs) Finance: The World Bank Key Messages Bulletin. Recuperado el 15 de mayo de 2018 de: http://www.worldbank.org/en/topic/smefinance

Banco Mundial. (2018). Enterprise Surveys 2017. Recuperado el 15 de noviembre de 2018 de: http://www.enterprisesurveys.org/data/exploreeconomies/2017/colombia

Baporikar, N., Nambira, G. y Gomxos, G. (2016). Exploring factors hindering SMEs' growth: evidence from Nambia. Journal of Science and Technology Policy Management, 7(2), 190-211. https://doi.org/10.1108/ JSTPM-11-2015-0036

Barbero, J., Casillas, J. y Feldman, H. (2011). Managerial capabilities and paths to growth as determinants of high-growth small and mediumsized enterprises. International Small Business Journal, 29(6), 671694. https://doi.org/10.1177/0266242610378287

Benzing, C., Chu, H. y Orhan, K. (2009). Entrepreneurs in Turkey: a factor analysis of motivations, success factors, and problems. Journal of Small Business Management, 47(1), 58-91. https://doi.org/10.1111/j.1540-627X.2008.00262.x

Blyde, J. (2014). Fábricas Sincronizadas: América Latina y el Caribe en la Era de las Cadenas Globales de Valor. Washington, DC: Banco Interamericano de Desarrollo. Recuperado el 15 de junio de 2018 de: https://publications.iadb.org/bitstream/handle/11319/6668/FLagship-2014-ESPANOL.pdf?sequence $=2$

Bouri, A., Breij, M., Diop, M., Kempner, R., Klinger, B. y Stevenson, K. (2011). Report on support to SMEs in Developing Countries through Financial Intermediaries. Recuperado el 10 de abril de 2018 de: http:// www.eib.org/attachments/dalberg_sme-briefing-paper.pdf

Burgstaller, J. y Wagner, E. (2015). How do family ownership and founder management affect capital structure decisions and adjustment of SMEs? Evidence from a bank-based economy. The Journal of Risk Finance, 16(1), 73-101. https://doi.org/10.1108/JRF-06-2014-0091

Calderón, G., Álvarez, C. y Naranjo, J. (2009). Orientación estratégica y recursos competitivos: Un estudio en grandes empresas industriales en Colombia. Cuadernos de Administración, 22(38), 49-72.

Candiya, G., Mpeera, J., Munene, J. y Akol, Ch. (2017). The relationship between access to finance and growth of SMEs in developing economies: Financial literacy as a moderator. Review of International Business and Strategy, 27(4), 520-538. https://doi.org/10.1108/RIBS-04-2017-0037

Casaburi, G., Suaznábar, C. y Llisterri, J. (2016). Extensión tecnológica para PyME. En: J. Navarro y Y. Olivari, (Eds), La política de innovación en América Latina y el Caribe. Nuevos caminos (pp. 101-132). Washington D.C: Banco Interamericano de Desarrollo.

Chatzoglou, P., Chatzoudes, D., Sarigiannidis, L. y Theriou, G. (2018). The role of firm-specific factors in the strategy-performance relationship: Revisiting the resource-based view of the firm and the VRIO framework. Management Research Review, 41(1), 46-73.

https://doi.org/10.1108/MRR-10-2016-0243

Correa, F., Dini, M. y Stumpo, G. (2018). El rol de las mipymes y las políticas de fomento: algunas conclusiones. En: M. Dini y G. Stumpo. (Coords.), MIPYMES en América Latina Un frágil desempeño y nuevos desafíos para las políticas de fomento (pp. 547-557). Santiago: Comisión Económica para América Latina y el Caribe (CEPAL).

Correa, F., Leiva, V. y Stumpo, G. (2018). Mipymes y heterogeneidad estructural en América Latina. En: M. Dini y G. Stumpo. (Coords.), MIPYMES en América Latina Un frágil desempeño y nuevos desafíos para las políticas de fomento (pp. 9-34). Santiago: Comisión Económica para América Latina y el Caribe (CEPAL).

Crespi, G., Tacsir, E. y Vargas, F. (2016). Innovation Dynamics and Productivity: Evidence for Latin America. En: M. Grazzi y C. Pietrobelli. (Eds.), Firm innovation and productivity in Latin America and the Caribbean. The Engine of Economic Development (pp. 37-71). New York: Springer Nature. https://doi.org/10.1057/978-1-349-58151-1

Departamento Administrativo Nacional de Estadística- DANE. (2016). Encuesta Anual Manufacturera- EAM. 2015. Recuperado el 22 de noviembre de 2018 de: https://www.dane.gov.co/index.php/estadis- ticas-por-tema/industria/encuesta-anual-manufacturera-enam/ eam-historicos

Departamento Administrativo Nacional de Estadística- DANE. (2017). Encuesta de Desarrollo e Innovación Tecnológica Industria manufacturera- EDIT VIII 2015-2016. Recuperado el 15 de noviembre de 2018 de: https:/www.dane.gov.co/files/investigaciones/boletines/ edit/boletin_EDIT_manufacturera_2015_2016.pdf

Departamento Nacional de Planeación de Colombia- DNP. (2016). CONPES 3866- Política nacional de desarrollo productivo. Recuperado el 20 de noviembre 2018 de: https://colaboracion.dnp.gov.co/ CDT/Conpes/Econ\%C3\%B3micos/3866.pdf

Dierickx, I. y Cool, K. (1989). Asset stock accumulation and sustainability of competitive advantage. Management Science, 35(12), 1504-1511. https://doi.org/10.1287/mnsc.35.12.1504

Dini, M. y Stumpo G. (2018). MIPYMES en América Latina Un frágil desempeño y nuevos desafíos para las políticas de fomento. Santiago: Comisión Económica para América Latina y el Caribe (CEPAL).

Drexler, A., Fischer, G. y Schoar, A. (2014). Keeping it simple: financial literacy and rules of thumb. American Economic Journal: Applied Economic, 6(2), 1-31. http://dx.doi.org/10.1257/app.6.2.1

Escandón-Barbosa, D., Arias, A., Medina, L., Gómez, L., Varela, R., Martínez, P. y Parra, L. (2016). Reporte GEM Colombia 2015/2016. Cali: Ediciones Sello Javeriano.

Franco, M., Santos, M., Ramalho, I. y Nunes, C, (2014). An exploratory study of entrepreneurial marketing in SMEs: The role of the founderentrepreneur. Journal of Small Business and Enterprise Development, 21(2), 265-283. https://doi.org/10.1108/JSBED-10-2012-0112

Franco-Ángel, M. y Urbano, D. (2010). El éxito de las PYMES en Colombia: un estudio de casos en el sector salud. Estudios Gerenciales, 26(114), 77-97. https://doi.org/10.1016/S0123-5923(10)70103-0

Franco-Ángel, M. y Urbano, D. (2014). El dinamismo de las PYMES en Colombia: un estudio comparativo de empresas. Revista Venezolana de Gerencia, 66(1), 319-338.

Franco-Ángel, M. y Urbano, D. (2016). Factores determinantes del dinamismo de las pequeñas y medianas empresas en Colombia. Revista de Ciencias Sociales, 22(1), 110-125.

Frohmann, A., Mulder, N. y Olmos, X. (2018). Promoción de la innovación exportadora Instrumentos de apoyo a las pymes. Santiago: Comisión Económica para América Latina y el Caribe (CEPAL).

Gómez, H. y Borda, S. (2018). Diagnóstico de las micro, pequeñas y medianas empresas: políticas e instituciones de fomento en Colombia. En M. Dini y G. Stumpo. (Coords.), MIPYMES en América Latina: Un frágil desempeño y nuevos desafíos para las políticas de fomento lpp. 227-288). Santiago: Comisión Económica para América Latina y el Caribe (CEPAL).

Grant, RM. (1991). The resource-based theory of competitive advantageImplications for strategy formulation. California Management Review, 33(3), 114-135. https://doi.org/10.2307/41166664

Haji, A. y Ghazali, N. (2018). The role of intangible assets and liabilities in firm performance: empirical evidence. Journal of Applied Accounting Research, 19(1), 42-59. https://doi.org/10.1108/JAAR-12-2015-0108

Hussain, J.G. y Matlay, H. (2007). Financing preferences of ethnic minority owner/managers in the UK. Journal of Business and Enterprise Development, 14(3), 487-500. https://doi.org/10.1108/14626000710773565

Jones, R. y Rowley, J. (2011). Entrepreneurial marketing in small businesses: A conceptual exploration. International Small Business Journal, 29(1) 25-36. https://doi.org/10.1177/0266242610369743

Kamasak, R. (2017). The contribution of tangible and intangible resources, and capabilities to a firm's profitability and market performance. European Journal of Management and Business Economics, 26(2), 252275. https://doi.org/10.1108/EJMBE-07-2017-015

Karlan, D. y Valdivia, M. (2011). Teaching entrepreneurship: impact of business training on microfinance institutions and clients. Review of Economics and Statistics, 93(2), 510-527. https://doi.org/10.1162/REST_a_00074

Kontinen, T. y Ojala, A. (2011). Network ties in the international opportunity recognition of family SMEs. International Business Review, 20(4), 440-453. https://doi.org/10.1016/j.ibusrev.2010.08.002

Kunc, M. y Morecroft, J. (2010). Managerial decision making and firm performance under a resource-based paradigm. Strategic Management Journal, 31(11), 1164-1182. https://doi.org/10.1002/smj.858

Lavia, O. y Hiebl, M. (2015). Management accounting in small and medi- 
um-sized enterprises: current knowledge and avenues for further research. Journal of Management Accounting Research, 27(1), 81-119. https://doi.org/10.2308/jmar-50915

Lindstrand, A., Melen, S. y Nordman, E. (2011). Turning social capital into business: a study of the internationalization of biotech SMEs. International Business Review, 20(2), 194-212. https://doi.org/10.1016/j.ibusrev.2011.01.002

Llisterri, J. y García-Alba, J. (2008). High-Growth SMEs in Latin American Emerging Economies. Recuperado el 20 de mayo de 2018 de: http://services.iadb.org/wmsfiles/products/Publications/35166777.pdf

Lussier, R. y Halabi, C. (2010). A three-country comparison of the business success versus failure prediction model. Journal of Small Business Management, 48(3), 360-377. https://doi.org/10.1111/j.1540-627X.2010.00298.x

Madrid-Guijarro, A., García-Pérez-de-Lema, D. y Van Auken, H. (2013). An Investigation of Spanish SME Innovation during Different Economic Conditions. Journal of Small Business Management, 51(4), pp. 578-601. https://doi.org/10.1111/jsbm.12004

Mendes, L. y Lourenço, L. (2014). Factors that hinder quality improvement programs' implementation in SME: Definition of a taxonomy. Journal of Small Business and Enterprise Development, 21(4), 690-715. https://doi.org/10.1108/JSBED-03-2014-0054

Middleton, K. y Chambers, V. (2010). Approaching digital equity: is wifi the new leveler? Information Technology \& People, 23(1), 4-22. https://doi.org/10.1108/09593841011022528

Ministerio de Comercio, Industria y Turismo (2018). Informe dinámicas de producción y comercio exterior de pymes manufactureras. Programa de Transformación Productiva. Recuperado el 1 de noviembre de 2018 de: https://www.ptp.com.co/CMSPages/GetFile. aspx?guid=1a3f1658-4ab6-432c-b038-6a2c7d9ad5a8

Murillo, M. y Restrepo, L. (2016). Nacimiento y supervivencia de las empresas en Colombia. Bogotá: Confecámaras. Recuperado el 1 de diciembre de 2018 de: http://www.confecamaras.org.co/phocadownload/Cuadernos_de_analisis_economico/Cuaderno_de_ An\%D0\%Bolisis_Economico_N_11.pdf

Presbitero, A. y Rabellotti, R. (2016). Credit Access in Latin American Enterprises. En M. Grazzi y C. Pietrobelli. (Eds.), Firm innovation and productivity in Latin America and the Caribbean. The Engine of Economic Development (pp. 245-284). New York: Springer Nature. https://doi.org/10.1057/978-1-349-58151-1

Rasmussen, P. y Nielsen, P. (2011). Knowledge management in the firm: concepts and issues. International Journal of Manpower, 32(5/6), 479493. https://doi.org/10.1108/01437721111158161

Schweizer, R. (2013). SMEs and networks: overcoming the liability of outsidership. Journal of International Entrepreneurship, 11(1), 80-103. https://doi.org/10.1007/s10843-012-0097-2

Segarra, A. y Callejón, M. (2000). New firm's survival and market turbulence. New evidence from Spain. Review of Industrial Organization, 20(1) 1-14.

Sidik, I. (2012). Conceptual framework of factors affecting SME development: mediating factors on the relationship of entrepreneur traits and SME performance. Proceedings from ICSMED 2012: International Conference on Small and Medium Enterprises. Procedia Economics and Finance, 4, 373-383.

https://doi.org/10.1016/S2212-5671(12)00351-6

Soini, E. y Veseli, L. (2011). Factors influencing SMEs growth in Kosovo (Tesis de Maestría). Recuperado el 10 de diciembre de 2018 de: https://www.theseus.fi/bitstream/handle/10024/28428/Soini_Eveliina_ Veseli_Labinot.pdf?sequ

Song, H. y Wang, L. (2013). The impact of private and family firms' relational strength on financing performance in clusters. Asia Pacific Journal of Management, 30(3), 735-748. https://doi.org/10.1007/s10490-012-9316-6

Spithoven, A., Vanhaverbeke, W. y Roijakkers, N. (2013). Open innovation practices in SMEs and large enterprises. Small Business Economics, 41(3), 537-562. https://doi.org/10.1007/s11187-012-9453-9

Swoboda, B., Meierer, M., Foscht, T. y Morschett, D. (2011). International SME alliances: the impact of alliance building and configurational fit on success. Long Range Planning, 44(4), 271-288.

https://doi.org/10.1016/j.lrp.2011.04.002

Tarapuez, E., Guzmán, B. y Parra, R. (2016). Estrategia e innovación en las mipymes colombianas ganadoras del Premio Innova 2010-2013. Estudios Gerenciales, 32(138), 170-180. http://dx.doi.org/10.1016/j.estger.2016.01.002

Temtime, Z. y Pansiri, J. (2005). Managerial competency and organizational flexibility in small and medium enterprises in Botswana. Problems and Perspectives in Management, 1, 25-36.

Tey, L. y Idris, A. (2012). Cultural fit, knowledge transfer and innovation performance: a study of Malaysian offshore international joint ventures. Asian Journal of Technology, 20(2), 201-218. https://doi.org/10.1080/19761597.2012.727698

Varum, C. y Rocha, V. (2013). Employment and SMEs during crises. Small Business Economics, 40(1), 9-25. https://doi.org/10.1007/s11187011-9343-6

Welter, F. y Smallbone, D. (2011). Institutional perspectives on entrepreneurial behaviour in challenging environments. Journal of Small Business Management, 49(1), 107-125. https://doi.org/10.1111/ j.1540-627X.2010.00317.x

Yan, S. (2010). Competitive strategy and business environment: the case of small enterprises in China. Asian Social Science, 6(11), 64-71. https://doi.org/10.5539/ass.v6n11p64 